\title{
A Numerical Method for Time-Fractional Reaction-Diffusion and Integro Reaction-Diffusion Equation Based on Quasi-Wavelet
}

\author{
Sachin Kumar, ${ }^{1}$ Jinde Cao $\mathbb{D}^{2},{ }^{2}$ and Xiaodi Li $\mathbb{i D}^{3}$ \\ ${ }^{1}$ Department of Mathematics, Govt. MGM PG College, Itarsi 461111, India \\ ${ }^{2}$ School of Mathematics, Southeast University, Nanjing 210096, China \\ ${ }^{3}$ School of Mathematics and Statistics, Shandong Normal University, Jinan 250014, China \\ Correspondence should be addressed to Jinde Cao; jdcao@seu.edu.cn
}

Received 16 May 2020; Accepted 4 August 2020; Published 26 September 2020

Academic Editor: Honglei Xu

Copyright ( $\odot 2020$ Sachin Kumar et al. This is an open access article distributed under the Creative Commons Attribution License, which permits unrestricted use, distribution, and reproduction in any medium, provided the original work is properly cited.

In this research work, we focused on finding the numerical solution of time-fractional reaction-diffusion and another class of integro-differential equation known as the integro reaction-diffusion equation. For this, we developed a numerical scheme with the help of quasi-wavelets. The fractional term in the time direction is approximated by using the Crank-Nicolson scheme. The spatial term and the integral term present in integro reaction-diffusion are discretized and approximated with the help of quasiwavelets. We study this model with Dirichlet boundary conditions. The discretization of these initial and boundary conditions is done with a different approach by the quasi-wavelet-based numerical method. The validity of this proposed method is tested by taking some numerical examples having an exact analytical solution. The accuracy of this method can be seen by error tables which we have drawn between the exact solution and the approximate solution. The effectiveness and validity can be seen by the graphs of the exact and numerical solutions. We conclude that this method has the desired accuracy and has a distinctive local property.

\section{Introduction}

Differential and integral calculus is a vital and broad branch of mathematics $[1,2]$. First of all, the integer-order differential and integer calculus came into existence. About a few decades ago, the concept of fractional calculus came into existence. Day by day the new concept, new definitions, and new terminology are emerging. The concepts and ideas of fractional calculus were evolved by Liouville and Abel. The more basic fundamentals and concepts are available in the literature $[3,4]$. This branch of mathematics allows us to generalize the order of derivatives from the integer order to the real and arbitrary order. There are so many types available in the literature of fractional calculus. Some of them are Caputo, Hadamard, Riemann-Liouville, and Grunwald-Letnikov. As time passes, in the development of this branch, the variable-order derivative concepts are introduced in which orders of derivatives are functions of variables. The most prevailing derivatives are power law kernel derivatives. But, in recent few years, the derivatives with nonsingular kernel are introduced. Derivatives with the Mittag-Leffler kernel are known as Atangana-Baleanu derivatives, and derivatives with the exponential kernel are known as the Caputo-Fabrizio derivative. The fractional differential equations are in trends to study. There are so many physical phenomena in which fractional differential equations depict and represent the behavior and dynamics of the phenomena in a more accurate way than integer-order differential equations. To find out the solution of the fractional differential equation and fractional partial differential equations is a tough task as it is not possible always to find out the exact solution. The researchers started to develop the methods and schemes for finding the numerical solution of fractional differential equations. Many numerical methods are developed and available in the literature. Some of them are the fractional differential transform method [5], predictor-corrector method [6], Adomian decomposition method [7], homotopy perturbation method [8], and generalized block pulse operational matrix method [9]. The operational matrix method is a spectral method which is 
easy to be applicable for many types of fractional differential equations whether they are of integer, fractional, and variable type. Its results are so accurate, and it is easy to apply. Some of them are Gegenbauer wavelet [9], Haar wavelets [10], Chebyshev wavelets [11], and Legendre wavelets [12]. The stability of discrete-time delayed switched systems and time delayed systems is given in the literature $[13,14]$.

FDEs, FPDEs, and fractional integro-differential equations are used to model many physical phenomena [15-17], which have arisen in physics, chemistry, biology, electrical engineering, nuclear reactor, viscoelastic, fluid dynamics, population dynamics, and medical science. To show the memory effect of the system, in the branch of nuclear reactor and thermo elasticity, we use the fractional differential equations. But, while modeling, the effect of history is being ignored. This can be taken into account by adding a term of integration in these fractional PDEs. The integro-differential equations have so many applications in control theory, financial market, fluid dynamics, aerospace systems, and viscoelastic mechanism. The most occurring physical process in nature is the diffusion process. In this process of diffusion, the molecules or energy or any physical quantity is transferred to the low concentration area from the high concentration area. If another process known as reaction is added to the process of diffusion, then it is called the reaction-diffusion process. In this process, molecules react with the fluids. This phenomenon of diffusion can be depicted by the following PDE known as the reaction-diffusion partial differential equation:

$$
\frac{\partial \Psi(\zeta, \eta)}{\partial \eta}=D \nabla^{2} \Psi(\zeta, \eta)+P(\Psi, \zeta, \eta)
$$

where $P(\Psi, \eta)$ represents the reaction term and the first term on the right-hand side of the equation represents the diffusion process with $D$ as the diffusion coefficient.

We divided our article into many sections as follows: Section 1 contains the preliminary definitions which will be used throughout the article. This section contains the theory and methods of the quasi-wavelet to find out an approximation of an unknown function. The taken model of integro reaction-diffusion and reaction-diffusion is given in Section 3. How to deal with discretization of spatial and timefractional terms with Dirichlet boundary conditions is also given in Section 3. The numerical results which depict the effectiveness and accuracy of our proposed method are present in Section 4. The conclusion of this paper is contained in Section 5.

\section{Preliminary Definition about Fractional Derivative and Quasi-Wavelets}

In this section, we described the definitions of fractional derivatives which will be used throughout the article. There are two types of definitions available in the literature of the fractional derivative, i.e., Riemann-Liouville and Caputo definitions. We have taken Caputo's one because it is more reliable in modeling.

2.1. Integration and Differentiation of Riemann-Liouville Type. The R-L-type definition of fractional integration is given as follows [18]:

$$
I^{\varrho} \zeta(\theta)=\frac{1}{\Gamma(\varrho)} \int_{0}^{\theta}(\theta-\Omega)^{\varrho^{-1}} \zeta(\Omega) \mathrm{d} \Omega, \quad \theta>0, \varrho \in R^{+} .
$$

The definition of fractional differentiation in the R-L sense is given by the following definition:

$$
D_{l}^{\varrho} \zeta(\theta)=\left(\frac{\mathrm{d}}{\mathrm{d} \theta}\right)^{m}\left(I^{m-\varrho} \zeta\right)(\theta),(\varrho>0, m-1<\varrho<m) .
$$

2.2. Definition of Fractional Derivative in Caputo Sense. The following equation represents the definition of fractional differentiation in Caputo sense:

$$
D_{c}^{\varrho} \zeta(\theta)= \begin{cases}\frac{\mathrm{d}^{q} \zeta(\theta)}{\mathrm{d} \theta^{q}}, & \varrho=q \in N, \\ \frac{1}{\Gamma(\varrho)} \int_{0}^{\theta}(\theta-\eta)^{q-\varrho-1} \zeta^{q}(\eta) \mathrm{d} \eta, & q-1<\varrho<q,\end{cases}
$$

where the parameter $q$ represents the integer. A resemblance of the Caputo definition with the integer-order derivative is as follows:

$$
D_{c}^{\varrho} M=0,
$$

with $M$ being a constant.

$$
D_{c}^{\varrho} \theta^{\sigma}= \begin{cases}0, & \sigma \in N \cup 0 \text { and } \sigma<\lceil\varrho\rceil, \\ \frac{\Gamma(1+\sigma)}{\Gamma(1-\varrho+\sigma)} \theta^{-\varrho+\sigma}, & \sigma \in N \cup 0 \text { and } \sigma \geq\lceil\varrho\rceil \text { or } \sigma \notin N \text { and } \sigma>\lceil\varrho\rceil,\end{cases}
$$

where $\lfloor\varrho\rfloor$ represents the floor function. All these types of fractional operators follow the linear property:

$$
D_{c}^{\varrho}\left(C_{1} h_{1}(\theta)+C_{2} h_{2}(\theta)\right)=C_{1} D_{c}^{\varrho} h_{1}(\theta)+C_{2} D_{c}^{\varrho} h_{2}(\theta),
$$

with $C_{1}$ and $C_{2}$ being constants. A useful relation between the R-L definition and Caputo is given as follows:

$$
\left(I^{9} D_{c}^{9} \varsigma\right)(\eta)=\varsigma(\eta)-\sum_{k=0}^{l-1} \varsigma^{k}\left(0^{+}\right) \frac{\eta^{k}}{k !}, \quad l-1<\vartheta \leq l .
$$


2.3. Approximation of Functions with the Help of QuasiWavelets. The method based upon quasi-wavelets nowadays is developing in the form of the local spectral collocation method. It is used for seeking the numerical solution of the fractional-order partial differential equation and integral equations. The singular convolution used in this method is a significant transformation. We define this transformation as follows:

$$
\varpi(\theta)=\left(R^{*} p\right)(\theta)=\int_{-\infty}^{\infty} R(\theta-\zeta) p(\zeta) \mathrm{d} \zeta
$$

where $p(\theta)$ is known as the test function and $R$ is a singular kernel. We know that the construction of the family of wavelets is done with the help of a function known as the mother wavelet $\varphi$. We use the operations of dilation and transformation to form the family of wavelets:

$$
\varphi_{\vartheta, \gamma}(\zeta)=\vartheta^{-1 / 2} \varphi\left(\frac{\zeta-\gamma}{\vartheta}\right)
$$

We have used two parameters here. The parameter $\vartheta$ is the dilation parameter, and $\gamma$ is the translation parameter. The kernel known as Shannon's delta sequence is used in the construction of quasi-wavelets. It is defined as

$$
\delta_{\theta}(\eta)=\frac{1}{\pi} \int_{0}^{\pi} \cos (\eta y) \mathrm{d} y=\frac{\sin (\theta \eta)}{\pi \eta},
$$

where $\lim _{\theta \longrightarrow \theta_{0}} \delta_{\theta}(\eta)=\delta(\eta)$ and $\delta$ is a well-known Dirac delta function. Shannon's delta sequence kernel produces the basis for the Paley-Wiener reproducing kernel Hilbert space $\mathbf{B}_{\theta}^{2}[19]$ for a given $\theta>0$ which is a subspace of $\mathbf{L}^{2}(R)$. We can produce uniquely a function $\varsigma(\zeta) \in \mathbf{B}_{\theta}^{2}$ as follows:

$$
\varsigma(\zeta)=\int_{-\infty}^{\infty} \varsigma(\zeta) \delta_{\theta}(\zeta-\eta) \mathrm{d} t=\int_{-\infty}^{\infty} \varsigma(\zeta) \frac{\sin (\theta(\zeta-\eta))}{\pi(\zeta-\eta)} \mathrm{d} \eta, \quad \forall \varsigma \in \mathbf{B}_{\theta}^{2}
$$

The sampling scaling function can be written in another form as follows:

$$
\delta_{\theta, p}=\delta_{\theta}\left(\zeta-\zeta_{p}\right)=\frac{\sin \left(\left(\zeta-\zeta_{p}\right) \theta\right)}{\left(\zeta-\zeta_{p}\right) \pi} .
$$

We have denoted the sampling points around the space $\zeta$ by $\left\{\zeta_{p}\right\}$ which are sampling points centered around $\zeta$. The discrete form of function $\varsigma(\zeta)$ which belongs to the space $\mathbf{B}_{\theta}^{2}$ is presented by the following infinite series expansion:

$$
\varsigma(\zeta)=\sum_{p=-\infty}^{\infty} \varsigma\left(\zeta_{p}\right) \delta_{\theta}\left(\zeta-\zeta_{p}\right)
$$

The above approximation can be rewritten with the help of Shannon sampling theorem at the Nyquist frequency $\theta$ in the band-limited signal in $B_{\theta}^{2}$ :

$$
\varsigma(\zeta)=\sum_{p=-\infty}^{\infty} \varsigma\left(\zeta_{p}\right) \delta_{\theta}\left(\zeta-\zeta_{p}\right)=\sum_{p=-\infty}^{\infty} \varsigma\left(\zeta_{p}\right) \frac{\sin \left(\pi\left(\zeta-\zeta_{p}\right) / \Delta\right)}{\left(\pi\left(\zeta-\zeta_{p}\right) / \Delta\right)},
$$

where the value of parameter $\theta$ is $\pi / \Delta$ and the grid size in spatial direction is denoted by $\Delta$. We improved the localized asymptotic behavior of Dirichlet's delta sequence kernel by using the regularizer $R_{\sigma}(\zeta)$. In the literature, many regularizers are introduced, and we use here the following Gaussian type regularizer:

$$
R_{\sigma}(\zeta)=\exp \left(\frac{-\zeta^{2}}{2 \sigma^{2}}\right), \quad \sigma>0,
$$

where $\sigma$ represents the width parameter of the Gaussian envelope. The parameters $\Delta$ and $\sigma$ are related by the expression $\sigma=r \times \Delta$, with $r$ as the computation parameter. The sampling scaling function is modified as follows by using the Gaussian regularizer:

$$
\delta_{\Delta, \sigma}(\zeta)=\frac{\sin (\pi \zeta / \Delta)}{\pi \zeta / \Delta} \exp \left(\frac{-\zeta^{2}}{2 \sigma^{2}}\right)
$$

where

$$
\lim _{\sigma \longrightarrow \infty} \delta_{\Delta, \sigma}(\zeta)=\frac{\sin (\pi \zeta / \Delta)}{(\pi \zeta / \Delta)}
$$

where the Gaussian-regularized sampling scaling function follows the property of the quasi-scaling function. So, we can approximate an unknown function $\varsigma(\zeta)$ belonging to the space textbf $B_{\theta}^{2}$ with the help of the quasi-scaling function:

$$
\varsigma(\zeta)=\sum_{p=-\infty}^{\infty} \varsigma\left(\zeta_{p}\right) \delta_{\theta}\left(\zeta-\zeta_{p}\right)=\sum_{p=-\infty}^{\infty} \varsigma\left(\zeta_{p}\right) \delta_{\theta}\left(\zeta-\zeta_{p}\right) R_{\theta}\left(\zeta-\zeta_{p}\right)
$$

Here, the infinite sampling points have been used, but while commutating in practical manner, we cannot use these infinite points. By seeing this problem, the domain of computation is restricted to the finite sampling points. For this purpose, we select $(2 W+1)$. The simplified form of the above equation is as follows:

$$
\varsigma(\zeta)=\sum_{p=-W}^{W} \varsigma\left(\zeta_{p}\right) \delta_{\Delta, \sigma}\left(\zeta-\zeta_{p}\right)
$$

Now, for the approximation of integer-order derivatives of order $n$ of the function $f(\zeta)$,

$$
\varsigma^{n}(\zeta)=\sum_{p=-W}^{W} \varsigma\left(\zeta_{p}\right) \delta_{\Delta, \sigma}^{n}\left(\zeta-\zeta_{p}\right), \quad n=1,2, \ldots
$$

The sampling points vary from $p=-W$ to $p=+W$, i.e., there are $2 W+1$ sampling points. We give some of the formula of $\delta_{\Delta, \sigma}, \delta_{\Delta, \sigma}^{1}$, and $\delta_{\Delta, \sigma}^{2}$ [20] for the calculation purpose: 


$$
\begin{aligned}
& \delta_{\Delta, \sigma}(\zeta)= \begin{cases}\frac{\exp \left\{-\zeta^{2} / 2 \sigma^{2}\right\} \sin (\zeta \pi / \Delta)}{(\pi \zeta / \Delta)}, & \zeta \neq 0 \\
1, & \zeta=0\end{cases} \\
& \delta_{\Delta, \sigma}^{1}(\zeta)= \begin{cases}\left(-\frac{\sin (\pi \zeta / \Delta)}{\left(\pi \zeta^{2} / \Delta\right)}-\frac{\Delta \sin (\pi \zeta / \Delta)}{\left(\pi \sigma^{2} / \Delta\right)}+\frac{\cos (\pi \zeta / \Delta)}{\zeta}\right) \exp \left(-\frac{\zeta^{2}}{2 \sigma^{2}}\right), & \zeta \neq 0, \\
0, & \zeta=0,\end{cases} \\
& \delta_{\Delta, \sigma}^{2}(\zeta)= \begin{cases}\left(\frac{2 \Delta \sin (\zeta \pi / \Delta)}{\pi \zeta^{3}}-\frac{2 \cos (\pi \zeta / \Delta)}{\zeta^{2}}+\frac{\Delta \zeta \sin (\zeta \pi / \Delta)}{\pi \sigma^{4}}+\frac{\Delta \sin (\pi \zeta / \Delta)}{\pi \sigma^{2} \zeta}-\frac{2 \cos (\pi \zeta / \Delta)}{\sigma^{2}}-\frac{\pi \sin (\zeta \pi / \Delta)}{\zeta \Delta}\right) \exp \left(-\frac{\zeta^{2}}{2 \sigma^{2}}\right), \quad \zeta \neq 0, \\
0, & \zeta=0 .\end{cases}
\end{aligned}
$$

\section{Mathematical Model and Used Proposed Method}

In this section, we propose the integrated mathematical model of the reaction-diffusion and integro reaction- diffusion equation. In this section, we develop the Crank-Nicolson scheme with the combination of the quasiwavelet-based numerical method to find the numerical solution of the taken model:

$$
\frac{\partial^{9} \varsigma}{\partial \eta^{9}}=\frac{\partial^{2} \varsigma}{\partial \zeta^{2}}+a \varsigma(\eta, \zeta)(1-\varsigma(\eta, \zeta))+b \varsigma(\eta, \zeta) \int_{0}^{\eta} \kappa(\eta-s, \zeta) \varsigma(s, \zeta) \mathrm{d} s+f(\zeta, \eta)
$$

We have taken the following Dirichlet boundary conditions and initial conditions:

$$
\varsigma(0, \eta)=g_{1}(\eta), \varsigma(1, \eta)=g_{2}(\eta), \varsigma(\zeta, 0)=g_{3}(\zeta),
$$

where the term $f(\zeta, \eta)$ denotes the forced function and $\kappa(\zeta, \eta)$ is known as a kernel.

$$
\begin{aligned}
\frac{\partial^{\vartheta} \varsigma\left(\zeta, \eta_{n+(1 / 2)}\right)}{\partial \eta^{9}}= & \frac{1}{\Gamma(1-\vartheta)} \int_{0}^{\eta_{n+1 / 2)}}\left(\eta_{n+(1 / 2)}-s\right)^{-\vartheta} \times \frac{\partial \varsigma(\zeta, s)}{\partial s} \mathrm{~d} s \\
= & \frac{1}{\Gamma(1-\vartheta)}\left[\int_{0}^{\eta_{n}}\left(\left(n+\frac{1}{2}\right) \Delta \eta-s\right)^{-\vartheta} \times \frac{\partial \varsigma\left(x_{i}, s\right)}{\partial s} \mathrm{~d} s+\int_{\eta_{n}}^{\eta_{\eta_{n+1 / 2}}}\left[\left(\frac{\varsigma^{n+1}-\varsigma^{n}}{\Delta \eta}\right)+O(\Delta \eta)\right]\left(\left(n+\frac{1}{2}\right) \Delta \eta-s\right)^{-\vartheta} \mathrm{d} s\right] \\
= & \frac{1}{\Gamma(1-\vartheta)} \sum_{m=1}^{n} \int_{(m-1) \Delta \eta}^{m \Delta \eta}\left(\left(\left(n+\frac{1}{2}\right) \Delta \eta-s\right)^{-\vartheta}\right) \times\left[\frac{\varsigma^{m+1}-\varsigma^{m}}{\Delta \eta}+\left(s-\eta_{m-(1 / 2)}\right) u_{t t}\left(c_{m}, \zeta\right)\right] \mathrm{d} s \\
& +\frac{1}{\Gamma(1-\vartheta)} \int_{n \Delta \eta}^{(n+(1 / 2)) \Delta \eta}\left[\left(\frac{\varsigma^{n+1}-\varsigma^{n}}{\Delta \eta}\right)+O(\Delta \eta)\right]\left(\left(n+\frac{1}{2}\right) \Delta \eta-s\right)^{-\vartheta} \mathrm{d} s .
\end{aligned}
$$

3.1. Using Crank-Nicolson Scheme for the Time Discretization. To discretize the time derivatives present in model, we will use the Crank-Nicolson scheme. We divided the time interval into discrete points as $\eta_{n}=n \times \Delta \eta$ where $n=0,1, \ldots, M$ and time length is noted by $\Delta \eta$. The notations $\varsigma^{n}$ and $f^{n}$ represent the values of $\varsigma(\zeta, \eta)$ and $f(\zeta, \eta)$ at time $\eta_{n}=n \times \Delta \eta$. The term $\partial^{\vartheta} \varsigma(\zeta, \eta) / \partial \eta^{\vartheta}$ from the model is approximated as at the grid point $\left(\zeta, \eta_{n+(1 / 2)}\right)$ : 
In aberration form, we can rewrite as

$$
\begin{aligned}
\frac{\partial^{9} \varsigma\left(\zeta, \eta_{n+(1 / 2)}\right)}{\partial \eta^{9}}= & \frac{\Delta \eta^{-\vartheta}}{\Gamma(2-\vartheta)} \sum_{m=1}^{n}\left(\varsigma^{m+1}-\varsigma^{m}\right)\left(\left(n+\frac{3}{2}-m\right)^{1-\vartheta}+\left(n+\frac{1}{2}-m\right)^{1-\vartheta}\right)+\frac{\Delta \eta^{-\vartheta}}{\Gamma(2-\vartheta)} \frac{\varsigma^{n+1}-\varsigma^{n}}{2^{1-9}}+\frac{1}{\Gamma(1-\vartheta)} \sum_{m=1}^{n} \int_{(m-1) \Delta \eta}^{m \Delta \eta} \\
& \cdot\left(\left(n+\frac{1}{2}\right) \Delta \eta-s\right)^{-\vartheta} \times\left(s-\eta_{m-(1 / 2)}\right) u_{t t}\left(c_{m}, \zeta\right) \mathrm{d} s+\frac{\Delta \eta^{-9}}{\Gamma(2-\vartheta)} \frac{1}{2^{1-\vartheta}} O\left((\Delta \eta)^{2-9}\right) .
\end{aligned}
$$

Hence, we obtain

$$
\frac{\partial^{\vartheta} \varsigma\left(x_{i}, \eta_{n+(1 / 2)}\right)}{\partial \eta^{9}}=\frac{\Delta \eta^{-\vartheta}}{\Gamma(2-\vartheta)}\left[\sum_{m=1}^{n}\left(u_{i}^{m+1}-u_{i}^{m}\right)\left(\left(n+\frac{3}{2}-m\right)^{1-\vartheta}+\left(n+\frac{1}{2}-m\right)^{1-\vartheta}\right)+\frac{u_{i}^{n+1}-u_{i}^{n}}{2^{1-9}}\right]+R_{1}+R_{2}
$$

where

$$
\begin{aligned}
& R_{1}=\frac{1}{\Gamma(1-\vartheta)} \sum_{m=1}^{n} \int_{(m-1) \Delta \eta}^{m \Delta \eta}\left(\left(\left(n+\frac{1}{2}\right) \Delta \eta-s\right)^{-\vartheta}\right) \times\left[\left(s-\eta_{m-(1 / 2)}\right) u_{t t}\left(c_{m}, x_{i}\right)\right] \mathrm{d} s, \\
& R_{2}=\frac{2^{9-1}}{\Gamma(2-\vartheta)} O\left((\Delta \eta)^{2-\vartheta}\right) .
\end{aligned}
$$

The bound of $R_{1}$ is as follows after simplifying:

$$
R_{1} \leq \frac{\max _{0 \leq \zeta \leq 1}\left|u_{t t}\left(c_{m}, \zeta\right)\right|}{\Gamma(1-\vartheta)} \Delta \eta^{2-\vartheta}
$$

The trapezoidal technique is implemented to deal with the integral term $\int_{0}^{\eta} \kappa(\eta-s, \zeta) \varsigma(s, \zeta) \mathrm{d} s$ :

$$
\int_{0}^{\eta_{n+1 / 2)}} \kappa(\zeta, \eta-s) \varsigma(\zeta, s) \mathrm{d} s=\frac{1}{2}\left\{\int_{0}^{\eta_{n}} \kappa(\zeta, \eta-s) \varsigma(\zeta, s) \mathrm{d} s+\int_{0}^{\eta_{n+1}} \kappa(\zeta, \eta-s) \varsigma(\zeta, s) \mathrm{d} s\right\} .
$$

Taking the exponential form of the kernel as $\kappa(\eta-s, \zeta)=e^{\{-(\eta-s)\}}$, then

$$
\begin{aligned}
\int_{0}^{\eta_{n}} \kappa(\zeta, \eta-s) \varsigma(\zeta, s) \mathrm{d} s & =\sum_{j=0}^{n-1} \int_{\eta_{j}}^{\eta_{j+1}} \exp \left\{-\left(\eta_{n}-s\right)\right\} \varsigma(\zeta, s) \mathrm{d} s \\
& =\sum_{j=0}^{n-1} \int_{\eta_{j}}^{\eta_{j+1}} \exp \left\{-\left(\eta_{n}-s\right)\right\}\left\{\varsigma\left(\zeta, \eta_{j+1}\right) \frac{s-\eta_{j}}{\Delta \eta}+\varsigma\left(\zeta, \eta_{j}\right) \frac{\eta_{j+1}-s}{\Delta \eta}\right\} \mathrm{d} s \\
& =\frac{e^{-\eta_{n}}}{\Delta \eta} \sum_{j=0}^{n-1}\left\{\varsigma^{j+1}\left(e^{\eta_{j+1}} \Delta \eta-e^{\eta_{j+1}}+e^{\eta_{j}}\right)+\varsigma^{j}\left(-e^{\eta_{j}} \Delta \eta+e^{\eta_{j+1}}-e^{\eta_{j}}\right)\right\} .
\end{aligned}
$$


Similarly,

$$
\begin{aligned}
\int_{0}^{\eta_{n+1}} \kappa(\zeta, \eta-s) \varsigma(\zeta, s) \mathrm{d} s= & \frac{e^{-\eta_{n+1}}}{\Delta \eta}\left\{\varsigma^{n+1}\left(e^{\eta_{n+1}} \Delta \eta-e^{\eta_{n+1}}+e^{\eta_{n}}\right)+\varsigma^{n}\left(-e^{\eta_{n}} \Delta \eta+e^{\eta_{n+1}}-e^{\eta_{n}}\right)\right\}+\frac{e^{-\eta_{n+1}}}{\Delta \eta} \sum_{j=0}^{n-1}\left\{\varsigma^{j+1}\left(e^{\eta_{j+1}} \Delta \eta-e^{\eta_{j+1}}+e^{\eta_{j}}\right)\right. \\
& \left.+\varsigma^{j}\left(-e^{\eta_{j}} \Delta \eta+e^{\eta_{j+1}}-e^{\eta_{j}}\right)\right\} .
\end{aligned}
$$

The temporal semidiscrete form is obtained with the help of equations (32)-(34):

$$
\begin{aligned}
& \frac{\Delta \eta^{-9}}{\Gamma(2-\vartheta)}\left[\sum_{m=1}^{n}\left(\varsigma^{m+1}-\varsigma^{m}\right)\left(\left(n+\frac{3}{2}-m\right)^{1-\vartheta}+\left(n+\frac{1}{2}-m\right)^{1-\vartheta}\right)+\frac{\varsigma^{n+1}-\varsigma^{n}}{2^{1-\vartheta}}\right] \\
& =b \varsigma^{n}(\zeta) \frac{e^{-\eta_{n}}+e^{-\eta_{n+1}}}{2 \Delta \eta} \sum_{j=0}^{n-1}\left\{\varsigma^{j+1}\left(e^{\eta_{j+1}} \Delta \eta-e^{\eta_{j+1}}+e^{\eta_{j}}\right)+\varsigma^{j}\left(-e^{\eta_{j}} \Delta \eta+e^{\eta_{j+1}}-e^{\eta_{j}}\right)\right\}+b \varsigma^{n}(\zeta) \frac{e^{-\eta_{n+1}}}{2 \Delta \eta}\left\{\varsigma^{n+1}\left(e^{\eta_{n+1}} \Delta \eta-e^{\eta_{n+1}}+e^{\eta_{n}}\right)\right. \\
& \left.\quad+\varsigma^{n}\left(-e^{\eta_{n}} \Delta \eta+e^{\eta_{n+1}}-e^{\eta_{n}}\right)\right\}+\frac{\partial^{2} \varsigma^{n}(\zeta)}{\partial \zeta^{2}}+a \varsigma^{n}(\zeta)\left(1-\varsigma^{n}(\zeta)\right) .
\end{aligned}
$$

3.2. Use of Quasi-Wavelet-Based Approximation for the Discretization of Spatial Derivatives. By using the approximation by quasi-wavelets given in Section 2, we discretize the unknown function and its spatial derivatives. The spatial step is chosen as $\Delta \zeta=1 / N$. We denote the value of the unknown function $\varsigma(\zeta, \eta)$ at spatial point $\zeta_{i}$ and time $\eta=t_{i}$ by $\varsigma_{i}^{n}$ with $i=0,1, \ldots, N$ and $n=0,1, \ldots, M$. By using the approximation from equation (21), we have

$$
\varsigma^{n}\left(x_{i}\right)=\sum_{p=i-W}^{i+W} \varsigma\left(\zeta_{k}, \eta_{n}\right) \delta_{\Delta, \sigma}^{n}\left(\zeta_{i}-\zeta_{k}\right), \quad n=0,1,2, \ldots, i=0,1, \ldots, N-1
$$

With the help of equation (33), we can rewrite equation (34) as follows:

$$
\begin{aligned}
& \frac{\Delta \eta^{-\vartheta}}{\Gamma(2-\vartheta)}\left[\sum_{m=1}^{n}\left(\varsigma^{m+1}-\varsigma^{m}\right)\left(\left(n+\frac{3}{2}-m\right)^{1-\vartheta}+\left(n+\frac{1}{2}-m\right)^{1-\vartheta}\right)+\frac{\varsigma^{n+1}-\varsigma^{n}}{2^{1-\vartheta}}\right] \\
& =b \sum_{k=i-W}^{i+W} \varsigma\left(\zeta_{k}, \eta_{n}\right) \delta_{\Delta, \sigma}\left(\zeta_{i}-\zeta_{k}\right) \frac{e^{-t \eta_{n}}+e^{-\eta_{n+1}}}{2 \Delta \eta} \sum_{j=0}^{n-1}\left\{\varsigma^{j+1}\left(e^{\eta_{j+1}} \Delta \eta-e^{\eta_{j+1}}+e^{\eta_{j}}\right)+\varsigma^{j}\left(-e^{\eta_{j}} \Delta \eta+e^{\eta_{j+1}}-e^{\eta_{j}}\right)\right\} \\
& \quad+b \sum_{p=i-W}^{i+W} \varsigma\left(\zeta_{k}, \eta_{n}\right) \delta_{\Delta, \sigma}\left(\zeta_{i}-\zeta_{k}\right) \frac{e^{-\eta_{n+1}}}{2 \Delta \eta} \\
& \left\{\varsigma^{n+1}\left(e^{\eta_{n+1}} \Delta \eta-e^{\eta_{n+1}}+e^{\eta_{n}}\right)+\varsigma^{n}\left(-e^{\eta_{n}} \Delta \eta+e^{\eta_{n+1}}-e^{\eta_{n}}\right)\right\}+\sum_{p=i-W}^{i+W} \varsigma\left(\zeta_{k}, \eta_{n}\right) \delta_{\Delta, \sigma}^{2}\left(\zeta_{i}-\zeta_{k}\right)+a \sum_{p=i-W}^{i+W} \varsigma\left(\zeta_{k}, \eta_{n}\right) \delta_{\Delta, \sigma}\left(\zeta_{i}-\zeta_{k}\right) \\
& \quad\left(1-\sum_{p=i-W}^{i+W} \varsigma\left(\zeta_{k}, \eta_{n}\right) \delta_{\Delta, \sigma}\left(\zeta_{i}-\zeta_{k}\right)\right) .
\end{aligned}
$$


Considering $x_{p}-x_{i}=x_{k}$, we have the following:

$$
\begin{aligned}
& \frac{\Delta \eta^{-9}}{\Gamma(2-\vartheta)}\left[\sum_{m=1}^{n}\left(\varsigma_{i}^{m+1}-\varsigma_{i}^{m}\right)\left(\left(n+\frac{3}{2}-m\right)^{1-\vartheta}+\left(n+\frac{1}{2}-m\right)^{1-9}\right)+\frac{\varsigma_{i}^{n+1}-\varsigma_{i}^{n}}{2^{1-9}}\right] \\
& =b \sum_{k=-W}^{+W} u_{k+i}^{n} \delta_{\Delta, \sigma}(-k \Delta \zeta) \times \frac{e^{-\eta_{n}}+e^{-\eta_{n+1}}}{2 \Delta \eta} \sum_{j=0}^{n-1}\left\{\zeta_{i}^{j+1}\left(e^{\eta_{j+1}} \Delta \eta-e^{\eta_{j+1}}+e^{\eta_{j}}\right)+\varsigma_{i}^{j}\left(-e^{\eta_{j}} \Delta \eta+e^{\eta_{j+1}}-e^{\eta_{j}}\right)\right\} \\
& \quad+b \sum_{k=-W}^{+W} u_{k+i}^{n} \delta_{\Delta, \sigma}(-k \Delta \zeta) \times \frac{e^{-\eta_{n+1}}}{2 \Delta \eta}\left\{\zeta_{i}^{n+1}\left(e^{\eta_{n+1}} \Delta \eta-e^{\eta_{n+1}}+e^{\eta_{n}}\right)+\zeta_{i}^{n}\left(-e^{\eta_{n}} \Delta \eta+e^{\eta_{n+1}}-e^{\eta_{n}}\right)\right\}+ \\
& \sum_{k=-W}^{+W} u_{k+i}^{n} \delta_{\Delta, \sigma}^{2}\left(\zeta_{i}-\zeta_{k}\right)+a \sum_{k=-W}^{i+W} u_{k+i}^{n} \delta_{\Delta, \sigma}(-k \Delta \zeta)\left(1-\sum_{k=-W}^{+W} u_{k+i}^{n} \delta_{\Delta, \sigma}(-k \Delta \zeta)\right) .
\end{aligned}
$$

This is the full discrete form of the taken model in case of the kernel $\kappa(\zeta, \eta)=e^{-\eta}$. For other kernels $\kappa(\zeta, \eta)=1$ and $\kappa(\zeta, \eta)=e^{-\zeta^{2} t}$, the discrete form is obtained as follows:

$$
\begin{aligned}
& \frac{\Delta \eta^{-\vartheta}}{\Gamma(2-\vartheta)}\left[\sum_{m=1}^{n}\left(\zeta_{i}^{m+1}-\zeta_{i}^{m}\right)\left(\left(n+\frac{3}{2}-m\right)^{1-\vartheta}+\left(n+\frac{1}{2}-m\right)^{1-\vartheta}\right)+\frac{\zeta_{i}^{n+1}-\zeta_{i}^{n}}{2^{1-9}}\right] \\
& =b\left(\frac{\Delta \eta}{4} \sum_{j=0}^{n-1}\left(u_{i}^{j}+\zeta_{i}^{j+1}\right)+\frac{\Delta \eta}{4} \sum_{j=0}^{n}\left(u_{i}^{j}+\zeta_{i}^{j+1}\right)\right)+\sum_{k=-W}^{+W} u_{k+i}^{n} \delta_{\Delta, \sigma}^{2}\left(\zeta_{i}-\zeta_{k}\right)+a \sum_{k=-W}^{i+W} u_{k+i}^{n} \delta_{\Delta, \sigma}(-k \Delta \zeta)\left(1-\sum_{k=-W}^{+W} u_{k+i}^{n} \delta_{\Delta, \sigma}(-k \Delta \zeta)\right), \\
& \quad \frac{\Delta \eta^{-9}}{\Gamma(2-\vartheta)}\left[\sum_{m=1}^{n}\left(\zeta_{i}^{m+1}-\zeta_{i}^{m}\right)\left(\left(n+\frac{3}{2}-m\right)^{1-\vartheta}+\left(n+\frac{1}{2}-m\right)^{1-\vartheta}\right)+\frac{\varsigma_{i}^{n+1}-\varsigma_{i}^{n}}{2^{1-\vartheta}}\right] \\
& =b \sum_{k=-W}^{+W} u_{k+i}^{n} \delta_{\Delta, \sigma}(-k \Delta \zeta) \times \frac{e^{-\eta_{n+1} x_{i}^{2}}}{2 \Delta \eta} \sum_{j=0}^{n-1}\left\{\zeta_{i}^{j+1}\left(\frac{e^{\eta_{j+1} x_{i}^{2}}}{x_{i}^{2}} \Delta \eta-\frac{e^{\eta_{j+1} x_{i}^{2}}}{x_{i}^{4}}+\frac{e^{\eta_{j} x_{i}^{2}}}{x_{i}^{4}}\right)+u_{i}^{j}\left(\frac{-e^{\eta_{j+1} x_{i}^{2}}}{x_{i}^{2}} \Delta \eta+\frac{e^{\eta_{j+1} x_{i}^{2}}}{x_{i}^{4}}-\frac{e^{\eta_{j} x_{i}^{2}}}{x_{i}^{4}}\right)\right\} \\
& \quad+\sum_{k=-W}^{+W} u_{k+i}^{n} \delta_{\Delta, \sigma}^{2}\left(\zeta_{i}-\zeta_{k}\right)+a \sum_{k=-W}^{i+W} u_{k+i}^{n} \delta_{\Delta, \sigma}(-k \Delta \zeta)\left(1-\sum_{k=-W}^{+W} u_{k+i}^{n} \delta_{\Delta, \sigma}(-k \Delta \zeta)\right) .
\end{aligned}
$$

The discretization of initial and boundary conditions is performed by adopting a technique because the value of $\varsigma\left(\zeta_{k}\right)$ is not defined outside the domain $[0,1]$ :

$$
\begin{aligned}
\varsigma(i, n) & =\varsigma_{i}^{n}=0, \quad i>M, i<0, n=0,1, \ldots, N, \\
u_{i}^{0} & =f_{3}\left(\zeta_{i}\right), \quad i=0,1, \ldots, M .
\end{aligned}
$$

3.3. Theorem (see [21]). Considering the function $f(\zeta)$ which is a member of the space $L_{\infty} \cap L_{2}(\Omega) \cap C^{s}(\Omega)$ and band limited to $B, s \in Z^{+}, \sigma=\Delta r, W \in N, W \geq r s / \sqrt{2}$. Then, we have the following required result:

$$
\left\|f^{s}-\sum_{k=-W}^{W} \delta_{\sigma, \Delta}^{s}\left(\zeta-\zeta_{k}\right) f\left(\zeta_{k}\right)\right\| \leq \beta \times \exp \left(\frac{-\gamma^{2}}{2 r^{2}}\right),
$$

where

$$
\begin{aligned}
& \gamma=\min \left(r^{2}(\pi-B \Delta), W\right), \\
& \beta=\left(\sqrt{2 B}\|f\|_{L_{s}(\Omega)}+2 r\|f\|_{L_{\infty}(\Omega)}\right) \times \frac{e^{\pi}(s+1) ! r}{\gamma \pi \Delta^{s}} .
\end{aligned}
$$




\section{Numerical Results and Validation}

In this section, we take some numerical examples of the reaction-diffusion equation and the integro reaction-diffusion equation. We show the validity and feasibility by showing the absolute error tables. The formula of finding errors $E_{r}$ between exact and numerical solutions is as follows:

$$
E_{r}=\left|\varsigma\left(\zeta_{i}, \eta_{j}\right)-\widetilde{\varsigma}\left(\zeta_{i}, \eta_{j}\right)\right|,
$$

where $\widetilde{\varsigma}\left(\zeta_{i}, \eta_{j}\right)$ denotes the numerical solution at point $\left(\zeta_{i}, \eta_{j}\right)$. We do all numerical computation in Wolfram Mathematica version-11.3.

Example 1. We take the following particular case of our model with parameters $a=1, \vartheta=0.9$, and $b=0$ :

$$
\frac{\partial^{9} \varsigma(\eta, \zeta)}{\partial \eta^{9}}=\frac{\partial^{2} \varsigma(\eta, \zeta)}{\partial \zeta^{2}}+\varsigma(\eta, \zeta)(1-\varsigma(\eta, \zeta))+f(\zeta, \eta)
$$

The initial and boundary conditions are taken as follows:

$$
\begin{array}{r}
\varsigma(\zeta, 0)=\sin (2 \pi \zeta), \\
\varsigma(0, \eta)=0, \varsigma(1, \eta)=0 .
\end{array}
$$

We determine the force function $f(\zeta, \eta)$ by putting the value of the exact solution $\varsigma(\zeta, \eta)=\sin (2 \pi \zeta) / \sqrt{\eta+1}$ in the taken particular case of the model. The graph is plotted for the exact and approximate solutions with parameters' value $M=30, \Delta \eta=0.0001$, and $N=10$ which can be seen in Figure 1. The variation of absolute errors between exact and numerical solutions is depicted in Table 1.

Example 2. Taking the following integro diffusion equation as a particular case of our model with parametric value $\kappa(\zeta, \eta)=1, a=0, \vartheta=1$, and $b=1$ :

$$
\frac{\partial \varsigma(\eta, \zeta)}{\partial \eta}=\frac{\partial^{2} \varsigma(\eta, \zeta)}{\partial \zeta^{2}}-\int_{0}^{\eta} \varsigma(s, \zeta) \mathrm{d} s+f(\zeta, \eta) .
$$

The boundary and initial conditions are taken as follows:

$$
\begin{aligned}
& \varsigma(0, \zeta)=\frac{1}{2}\left(1-\zeta^{2}\right), \\
& \varsigma(\eta, 0)=\frac{\cosh (\eta)}{\sinh ^{2}(\eta)+2}, \\
& \varsigma(\eta, 1)=0 .
\end{aligned}
$$

The exact solution of the above problem is taken as $\varsigma(\zeta, \eta)=\left(1-\zeta^{2}\right) \cosh (\eta) / \sinh ^{2}(\eta)+2$ while force function $f(\zeta, \eta)$ is chosen with the help of exact solutions and the given problem. The graph is plotted for the numerical and exact solutions with parameters' value $N=10, M=30$, and $\Delta \eta=0.0001$ which can be seen in Figure 2 . The variation of absolute errors between exact and numerical solutions is depicted in Table 2.

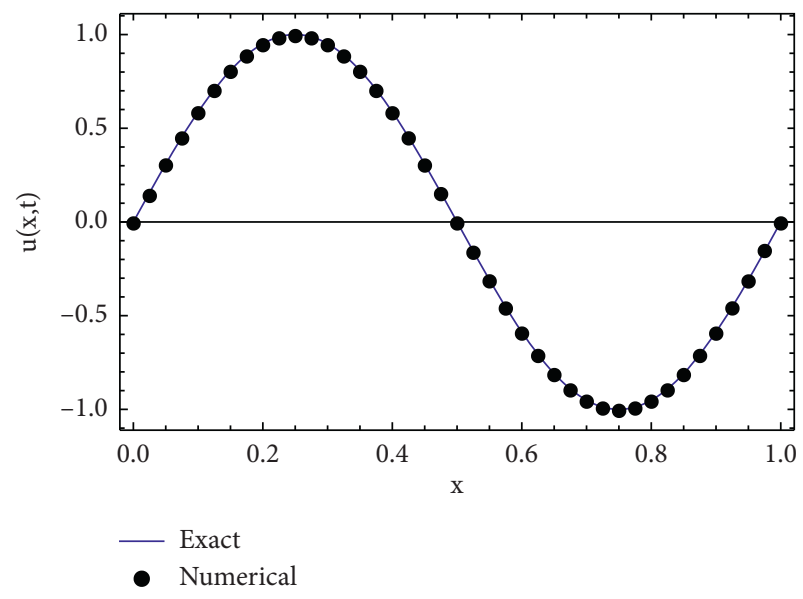

FIgURE 1: Graph of exact and numerical solutions with parametric value $\varsigma(\zeta, \eta)$ for $M=30, \vartheta=0.9, W=30, \Delta \eta=0.00001$, and $r=3.2$.

TABLE 1: Representation of absolute errors for different $M$ and $\Delta \eta$.

\begin{tabular}{lcc}
\hline $\mathrm{M} \downarrow$ & $\Delta \eta=0.0001$ & $\Delta \eta=0.000001$ \\
\hline 10 & $6.50 \times 10^{-2}$ & $3.6 \times 10^{-3}$ \\
15 & $4.5 \times 10^{-2}$ & $1.1 \times 10^{-3}$ \\
20 & $7.60 \times 10^{-3}$ & $1.15 \times 10^{-4}$ \\
30 & $9.10 \times 10^{-4}$ & $2.2 \times 10^{-5}$ \\
\hline
\end{tabular}

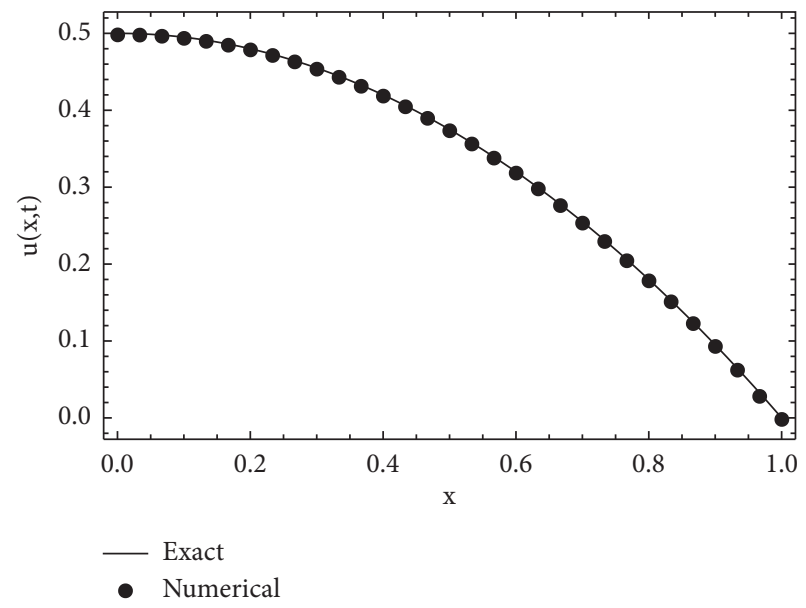

FIGURE 2: Graph of approximate and exact and numerical solutions with parametric value $\varsigma(\zeta, \eta)$ for $M=30, \vartheta=1, \quad W=30$, $\Delta \eta=0.00001$, and $r=3.2$.

TABLE 2: Representation of absolute errors for different $M$ and $\Delta \eta$.

\begin{tabular}{lcc}
\hline $\mathrm{M} \downarrow$ & $\Delta \eta=0.0001$ & $\Delta \eta=0.000001$ \\
\hline 10 & $9.40 \times 10^{-4}$ & $3.4 \times 10^{-5}$ \\
15 & $7.1 \times 10^{-4}$ & $2.4 \times 10^{-5}$ \\
20 & $4.6 \times 10^{-4}$ & $1.9 \times 10^{-5}$ \\
30 & $4.0 \times 10^{-4}$ & $1.3 \times 10^{-5}$ \\
\hline
\end{tabular}


Example 3. If we take $a=1, \vartheta=1, b=1$, and kernel $\kappa(\eta, \zeta)=e^{-\zeta^{2} t}$, then we get

$$
\begin{gathered}
\frac{\partial \varsigma(\eta, \zeta)}{\partial \eta}=\frac{\partial^{2} \varsigma(\eta, \zeta)}{\partial \zeta^{2}}+\varsigma(\eta, \zeta)(1-\varsigma(\eta, \zeta))+\varsigma(\eta, \zeta) \\
\int_{0}^{\eta} e^{-\zeta^{2}(\eta, \zeta)} \varsigma(\eta, \zeta) \mathrm{d} s+f(\eta, \zeta) .
\end{gathered}
$$

With the following initial and boundary conditions:

$$
\varsigma(\zeta, 0)=0, \varsigma(0, \eta)=\sin \eta, \varsigma(1, \eta)=\frac{\sin \eta}{2} .
$$

The function $\varsigma(\zeta, \eta)=\sin \eta / 1+\zeta^{2}$ is an exact solution of the problem. The graph is plotted for the numerical and exact solutions with parameters' value $N=10, M=30$, and $\Delta \eta=0.0001$ which can be seen in Figure 3 . The variation of absolute errors between exact and numerical solutions is depicted in Table 3 .

Example 4. If we take $\kappa(\eta, \zeta)=e^{-\eta}, a=1, \vartheta=0.9$, and $b=1$, then our model (42) is reduced to

$$
\begin{gathered}
\frac{\partial^{0.9} \varsigma(\eta, \zeta)}{\partial \eta^{0.9}}=\frac{\partial^{2} \varsigma(\eta, \zeta)}{\partial \zeta^{2}}+\varsigma(\eta, \zeta)(1-\varsigma(\eta, \zeta))+\varsigma(\eta, \zeta) \\
\int_{0}^{\eta} e^{-(\eta-s)} \varsigma(s, \zeta) \mathrm{d} s+f(\zeta, \eta) .
\end{gathered}
$$

The following equations are chosen as initial and boundary conditions:

$$
\begin{aligned}
& \varsigma(\zeta, 0)=\left(1-\zeta^{4}\right) \sin (\zeta), \\
& \varsigma(0, \eta)=\sin \eta, \\
& \varsigma(1, \eta)=0,
\end{aligned}
$$

with the exact solution $\varsigma(\zeta, \eta)=\sin (\eta+\zeta)\left(1-\zeta^{4}\right)$.

The graph is plotted for the exact and approximate solutions with parameters' value $N=10, M=30$, and $\Delta \eta=$ 0.0001 which can be seen in Figure 4. The variation of absolute errors between exact and numerical solutions is depicted in Table 4 . The graphs and error tables clearly show that our numerical results are in feasible and desirable accuracy.

Example 5. Let us take another example of the BurgerFisher equation:

$$
\frac{\partial \varsigma(\zeta, \eta)}{\partial \eta}=\frac{\partial^{2} \varsigma(\zeta, \eta)}{\partial \zeta^{2}}+\varsigma \frac{\partial \varsigma(\zeta, \eta)}{\partial \zeta}+\varsigma(1-\varsigma), \quad 0<\zeta<1, \eta>0,
$$

with following initial and boundary conditions:

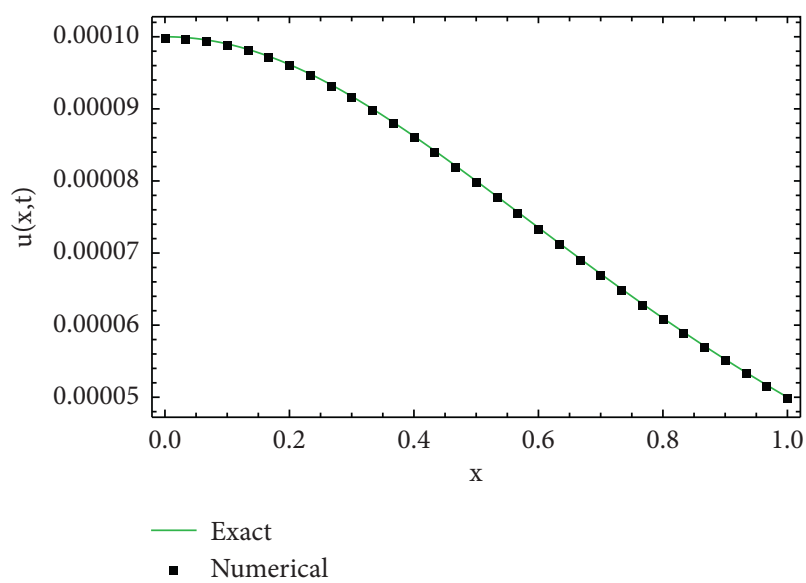

FIGURE 3: Graph of approximate and exact solutions with parametric value $\varsigma(\zeta, \eta)$ for $\vartheta=1, W=30, \Delta \eta=0.00001, M=30$, and $r=3.2$.

TABLE 3: Representation of absolute errors for different $M$ and $\Delta \eta$.

\begin{tabular}{lcc}
\hline $\mathrm{M} \downarrow$ & $\Delta \eta=0.0001$ & $\Delta \eta=0.000001$ \\
\hline 10 & $1.97 \times 10^{-7}$ & $7.4 \times 10^{-13}$ \\
15 & $1.90 \times 10^{-7}$ & $4.8 \times 10^{-13}$ \\
20 & $1.84 \times 10^{-7}$ & $3.5 \times 10^{-13}$ \\
30 & $1.7 \times 10^{-7}$ & $2.2 \times 10^{-13}$ \\
\hline
\end{tabular}

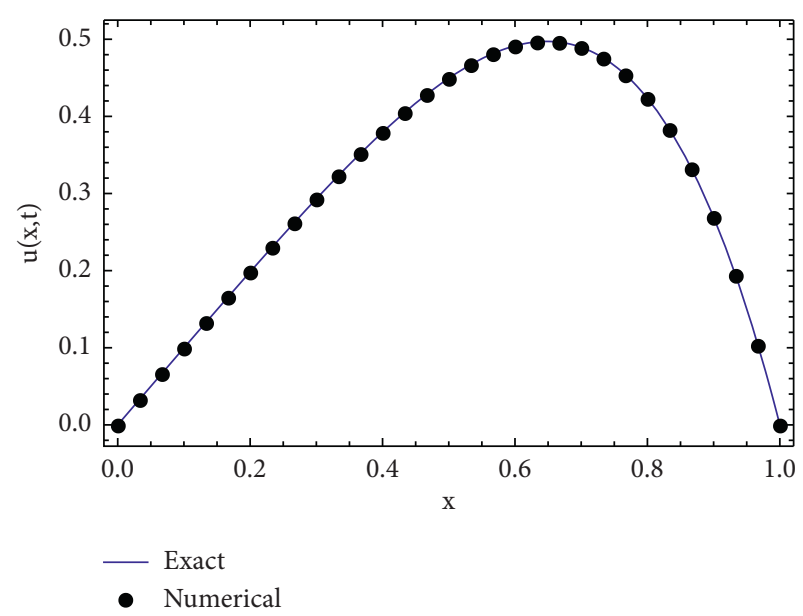

Figure 4: Graph of approximate and exact solutions with parametric value $\varsigma(\zeta, \eta)$ for $M=30, \vartheta=0.9, W=30, \Delta \eta=0.00001$, and $r=3.2$.

TABLE 4: Representation of absolute errors for different $M$ and $\Delta \eta$.

\begin{tabular}{lcc}
\hline $\mathrm{M} \downarrow$ & $\Delta \eta=0.0001$ & $\Delta \eta=0.000001$ \\
\hline 10 & $9.80 \times 10^{-4}$ & $4.9 \times 10^{-5}$ \\
15 & $5.5 \times 10^{-4}$ & $3.3 \times 10^{-5}$ \\
20 & $2.90 \times 10^{-4}$ & $2.5 \times 10^{-5}$ \\
30 & $1.50 \times 10^{-4}$ & $1.7 \times 10^{-5}$ \\
\hline
\end{tabular}


TABLE 5: Comparison of absolute errors for our proposed method and the method given in [22].

\begin{tabular}{ccc}
\hline$(\zeta, \eta) \downarrow$ & Our method with $\Delta \eta=10^{-7}$ & Method given in literature \\
\hline$(0.1,1)$ & $6,9 \times 10^{-8}$ & $2.51 \times 10^{-7}$ \\
$(0.2,1)$ & $5.9 \times 10^{-8}$ & $1.07 \times 10^{-6}$ \\
$(0.3,1)$ & $3.5 \times 10^{-8}$ & $2.13 \times 10^{-6}$ \\
$(0.4,1)$ & $6.5 \times 10^{-8}$ & $3.21 \times 10^{-6}$ \\
$(0.5,1)$ & $5.7 \times 10^{-8}$ & $4.13 \times 10^{-6}$ \\
$(0.6,1)$ & $4.9 \times 10^{-8}$ & $4.74 \times 10^{-6}$ \\
$(0.7,1)$ & $8.2 \times 10^{-7}$ & $4.89 \times 10^{-6}$ \\
$(0.8,1)$ & $1.5 \times 10^{-8}$ & $4.37 \times 10^{-6}$ \\
$(0.9,1)$ & $4.40 \times 10^{-7}$ & $2.8 \times 10^{-6}$ \\
\hline
\end{tabular}

$$
\begin{aligned}
& \varsigma(\zeta, 0)=\frac{1}{2}+\frac{1}{2} \tanh \left(\frac{\zeta}{4}\right), \\
& \varsigma(0, \eta)=\frac{1}{2}+\frac{1}{2} \tanh \left(\frac{5 \eta}{8}\right), \\
& \varsigma(1, \eta)=\frac{1}{2}+\frac{1}{2} \tanh \left(\frac{1}{4}\left(1+\frac{5 \eta}{2}\right)\right),
\end{aligned}
$$

where the exact solution of the above problem is $(1 / 2)+(1 / 2) \tanh ((1 / 4)(\zeta+5 \eta / 2))$. Now, our obtained numerical results are compared with the results given in [22].

\section{Conclusion}

In this research work, we developed a numerical scheme for time-fractional partial differential equations and integro fractional partial differential equations. The discretization of the time-fractional derivative is done by using the Crank-Nicolson scheme, and the discretization of the unknown function and its spatial derivatives is performed with the help of the quasi-wavelet-based numerical method. For different kernels, we show the validity and effectiveness of our method. Our method has a good accuracy for both fractional PDEs and fractional integro PDEs as seen from the error tables. The same can be seen by the plotted graph for the existing exact solution and numerical solution.

\section{Data Availability}

No data were used to support this study.

\section{Conflicts of Interest}

The authors declare that there are no conflicts of interest.

\section{Acknowledgments}

This work was supported by the National Natural Science Foundation of China (61673247) and the Research Fund for Distinguished Young Scholars and Excellent Young Scholars of Shandong Province (JQ201719).

\section{References}

[1] X. Lv, R. Rakkiyappan, R. Rakkiyappan, and X. Li, "m-stability criteria for nonlinear differential systems with additive leakage and transmission time-varying delays," Nonlinear Analysis: Modelling and Control, vol. 23, no. 3, pp. 380-400, 2018.

[2] A. Vinodkumar, T. Senthilkumar, and X. Li, "Robust exponential stability results for uncertain infinite delay differential systems with random impulsive moments," Advances in Difference Equations, no. 1, p. 39, 2018.

[3] A. Kilbas, H. Srivastava, and J. J. Trujillo, Theory and Applications of the Fractional Differential Equations, Vol. 204, Elsevier, Amsterdam, Netherlands, 2006.

[4] I. Podlubny, Fractional Differential Equations, to Methods of Their Solution and Some of Their Applications, Fractional Differential Equations: An Introduction to Fractional Derivatives, Academic Press, San Diego, CA, USA, 1998.

[5] P. Darania and A. Ebadian, "A method for the numerical solution of the integro-differential equations," Applied Mathematics and Computation, vol. 188, no. 1, pp. 657-668, 2007.

[6] K. Diethelm, N. J. Ford, and A. D. Freed, "A predictor-corrector approach for the numerical solution of fractional differential equations," Nonlinear Dynamics, vol. 29, no. 1-4, pp. 3-22, 2002.

[7] L. Suarez and A. Shokooh, "An eigenvector expansion method for the solution of motion containing fractional derivatives," Journal of Applied Mechanics, vol. 64, p. 9, 1997.

[8] S. Kumar and D. Baleanu, "Numerical solution of two-dimensional time fractional cable equation with mittag-leffler kernel," Mathematical Methods in the Applied Sciences, vol. 43, no. 15, pp. 8348-8362, 2020.

[9] S. Kumar, P. Pandey, and S. Das, "Gegenbauer wavelet operational matrix method for solving variable-order non-linear reaction-diffusion and galilei invariant advection-diffusion equations," Computational and Applied Mathematics, vol. 38, no. 4, p. 162, 2019.

[10] Y. Li and W. Zhao, "Haar wavelet operational matrix of fractional order integration and its applications in solving the fractional order differential equations," Applied Mathematics and Computation, vol. 216, no. 8, pp. 2276-2285, 2010.

[11] L. Yuanlu, "Solving a nonlinear fractional differential equation using Chebyshev wavelets," Communications in Nonlinear Science and Numerical Simulation, vol. 15, no. 9, pp. 2284-2292, 2010.

[12] H. Jafari, S. A. Yousefi, M. A. Firoozjaee, S. Momani, and C. M. Khalique, "Application of legendre wavelets for solving fractional differential equations," Computers \& Mathematics with Applications, vol. 62, no. 3, pp. 1038-1045, 2011.

[13] B. Liu and D. J. Hill, "Stability via hybrid-event-time Lyapunov function and impulsive stabilization for discrete-time delayed switched systems," SIAM Journal on Control and Optimization, vol. 52, no. 2, pp. 1338-1365, 2014.

[14] B. Liu, D. J. Hill, Z. Sun, and J. Huang, "Event-triggered control via impulses for exponential stabilization of discretetime delayed systems and networks," International Journal of Robust and Nonlinear Control, vol. 29, no. 6, pp. 1613-1638, 2019.

[15] X. Li, X. Yang, and T. Huang, "Persistence of delayed cooperative models: impulsive control method," Applied Mathematics and Computation, vol. 342, pp. 130-146, 2019.

[16] D. Yang, X. Li, J. Shen, and Z. Zhou, "State-dependent switching control of delayed switched systems with stable and 
unstable modes," Mathematical Methods in the Applied Sciences, vol. 41, no. 16, pp. 6968-6983, 2018.

[17] X. Zhang, X. Li, and X. Han, "Design of hybrid controller for synchronization control of chen chaotic system," The Journal of Nonlinear Sciences and Applications, vol. 10, no. 6, pp. 3320-3327, 2017.

[18] D. Tavares, R. Almeida, and D. F. M. Torres, "Caputo derivatives of fractional variable order: numerical approximations," Communications in Nonlinear Science and Numerical Simulation, vol. 35, pp. 69-87, 2016.

[19] G. W. Wei, "Discrete singular convolution for the solution of the Fokker-Planck equation," The Journal of Chemical Physics, vol. 110, no. 18, pp. 8930-8942, 1999.

[20] X. Yang, D. Xu, and H. Zhang, "Quasi-wavelet based numerical method for fourth-order partial integro-differential equations with a weakly singular kernel," International Journal of Computer Mathematics, vol. 88, no. 15, pp. 32363254, 2011.

[21] L. Qian, "On the regularized Whittaker-Kotel'nikov-Shannon sampling formula," Proceedings of the American Mathematical Society, vol. 131, no. 4, pp. 1169-1176, 2003.

[22] S. Kumar, P. Pandey, and S. Das, "Operational matrix method for solving nonlinear space-time fractional order reactiondiffusion equation based on genocchi polynomial," Special Topics \& Reviews in Porous Media: An International Journal, vol. 11, no. 1, pp. 33-47, 2020. 